\title{
SIMULATION OF QUASISTATIONARY ELECTROMAGNETIC FIELDS IN REGIONS CONTAINING DISCONNECTED CONDUCTING SUBREGIONS
}

\author{
M.P. Galanin ${ }^{1,2}$ \\ D.L. Sorokin ${ }^{1}$
}

galan@keldysh.ru

sorokin.dmitr@yandex.ru

\author{
${ }^{1}$ Keldysh Institute of Applied Mathematics, Russian Academy of Sciences, \\ Moscow, Russian Federation \\ ${ }^{2}$ Bauman Moscow State Technical University, Moscow, Russian Federation
}

\begin{abstract}
Methods for a numerical solution of Maxwell's equations in the quasistationary aproximation in a region with multiply connected conducting subregions were discussed. The case of nontrivial operator kernel was explored. The methods for finding the solution of the linear algebraic equations system were analyzed. The method of introducing a "fictional armature" was offered as alternative method for searching [retrieving] a normal solution of linear algebraic equations. Results of computational experiments were presented. The study was carried out on the example of calculation for electrodynamic acceleration process in the railgun channel
\end{abstract}

\section{Keywords}

Maxwell's equations, normal solution, electrodynamic accelerator, railgun, augmenting turn

Received 12.01.2018

(C) Author(s), 2019

The work was carried out with the state financial support of the RFBR (grant no. 18-01-00252)

Introduction. The relatively slow electrodynamic processes, the characteristic time of change of which is much longer than the time of light passing through the characteristic spatial scale of the task, are considered. The conductivity of materials through which the electric current flows is considered to be rather high, so that the conditions for the applicability of the magnetohydrodynamic (in this case, quasistationary) approximation of Maxwell's equations are satisfied. Another feature of this problem formulation is that the processes occurring in the conductive parts are of the main interest.

As shown in [1], to describe these phenomena, the quasistationary approximation of Maxwell's equations can be used, although its applicability in the whole region is not provided by the materials parameters. In this case, the solution 
Simulation of Quasistationary Electromagnetic Fields in Regions...

for the used system is not unique. Both electric and magnetic fields are determined uniquely in the conducting subregion. The magnetic field is the only one in a nonconducting subregion. The system of equations of the quasistationary approximation in [1] is modified so that the uniqueness of the modified system solution in the region is provided if the conducting subregion with a part of the certain type boundary is a connected domain. If it is not, then additional problems arise with ensuring the uniqueness of the solution.

This work is devoted to the numerical solution of these tasks. The electrodynamic accelerators of the railgun macro-type are considered [1]. Calculated regions with disconnected conducting subregions arise, in particular, when simulating the macro-body acceleration in a railgun channel with augmenting rails.

The problem formulation. Mathematical model. The scheme of the simplest railgun channel is shown at Fig. 1. A power supply is connected to the conducting rails. Between the rails there is a movable projectile, which closes the circuit, as a result the electric current flows along the rails and projectile. A magnetic field arises around the rails. The interaction of the field and the electric current in the projectile leads to the genaration of the Lorentz (Ampere) force, which pushes the projectile out of the accelerator channel $[2,3]$.
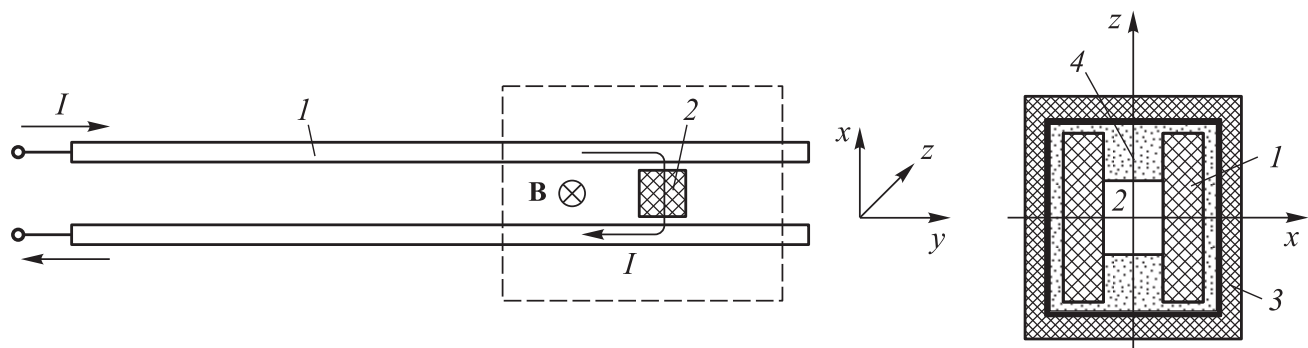

Fig. 1. Scheme of the simplest railgun channel:

1 - guide rail and conducting rail; 2 - accelerated body (projectile or other armature); 3 - railgun power bandage; 4 - isolator

The design of an accelerator can be complicated by introducing a augmenting [4-6], an armature shape meshing [7], combining several simple accelerators into one multi-turn [8-10], and so on.

The most important phenomena in electrodynamic accelerators occur in the vicinity of the armature. The electrical conductivity of the material of the rails and the projectile is rather high, so the electromagnetic field in these elements can be described by the system of Maxwell equations in the magnetohydrodynamic (MHD) approximation $[1,11]$. However, the computational domain contains not 
M.P. Galanin, D.L. Sorokin

only conductors, but also dielectrics. In [1], it was shown that in order to find a unique solution in a dielectric, the system of Maxwell equations in the MHD approximation had to be supplemented with the condition that the electric field strength in the dielectric was equal to zero and special boundary conditions.

The the task statement for determining the electromagnetic fields inside the region after the the vector potential $\mathbf{A}$ introduction [12]:

$$
\begin{gathered}
\left\{\begin{array}{l}
\mathbf{H}=\operatorname{rot} \mathbf{A} ; \\
\mathbf{E}=[\mathbf{u} \times \operatorname{rot} \mathbf{A}]-\frac{D \mathbf{A}}{D t}+(\mathbf{v} \cdot \nabla \mathbf{A}) ;
\end{array}\right. \\
\left\{\begin{array}{l}
4 \pi \sigma\left\{[\mathbf{u} \times \operatorname{rot} \mathbf{A}]-\frac{D \mathbf{A}}{D t}+(\mathbf{v}, \nabla) \mathbf{A}\right\}=\operatorname{rot} \operatorname{rot} \mathbf{A}-\theta(\sigma) \operatorname{grad} \operatorname{div} \mathbf{A} ; \\
\left.(\operatorname{rot} \mathbf{A})_{\tau}\right|_{r \in \Gamma_{2}}=\Psi_{\tau}(\mathbf{r}, t),\left.\quad \mathbf{A}_{\tau}\right|_{\mathbf{r} \in \Gamma_{1}}=0,\left.\operatorname{div} \mathbf{A}\right|_{\mathbf{r} \in \gamma_{12}}=0,\left.A_{n}\right|_{\mathbf{r} \in \Gamma_{22}}=0 ; \\
\left.\mathbf{A}\right|_{t=0, \mathbf{r} \in G_{1}}=0 .
\end{array}\right.
\end{gathered}
$$

Here $\mathbf{H}$ is magnetic field vector; $\mathbf{E}$ is electric field vector in the coordinate system in which the substance is in the state of rest; $\mathbf{u}$ is substance velocity; $\mathbf{v}$ is the speed of the rigid projectile; $t$ is time; $\sigma$ is conductivity; $\mathbf{r}=$ $=(x, y, z)$ is radius-vector; $G$ is considered domain $\left(G=G_{1} \cup G_{2}\right) ; G_{1}=$ $=\{\mathbf{r} \in G: \sigma>0\}$ is conductor; $G_{2}=\{\mathbf{r} \in G: \sigma=0\}$ is dielectric; $\partial G_{1}, \partial G_{2}$ are $G_{1}$ and $G_{2}$ boundaries; $\partial G_{12}=\partial G_{1} \cap \partial G_{2} ; \Gamma_{1}$ is part of the overall boundary $\partial G$, on which there is condition on $\mathbf{A}_{\tau}$ (index $\tau$ indicates tangential component); $\Gamma_{2}$ is part of the overall boundary $\partial G$, on which there is condition on $(\operatorname{rot} \mathbf{A})_{\tau}$ $\left(\Psi_{\tau} \quad\right.$ is known vector function); $\partial G=\Gamma_{1} \cup \Gamma_{2}, \quad \Gamma_{12}=\Gamma_{1} \cap \partial G_{2}, \quad \Gamma_{22}=$ $=\Gamma_{2} \cap \partial G_{2}, \quad \gamma_{12}=\partial G_{12} \cup \Gamma_{12}$. The space discontinuity is taken into account: $\theta(\sigma)=0$ in $G_{1}$ and $\theta(\sigma)=1$ in $G_{2}$.

The finite difference method was used for solving the system of equations. The method of basic operators $[1,12,13]$ was used for a difference scheme construction.

Let's denote the operator applied to $\mathbf{A}$ by $\mathcal{M}$ and the matrix of a system of difference linear algebraic equations by $M$, then the system without taking into account the initial values can be briefly written as $\mathcal{M A}=\mathbf{f}$ and the corresponding difference task - as $M A=f$.

The features of the task formulation in the presence of augmenting turns. The operator kernel. The magnetization system is a set of conductors (hereinafter - augmenting rails) through which an electric current flows that is different from the current in the guides. In this case the power supply 
Simulation of Quasistationary Electromagnetic Fields in Regions...

of the magnetization system is conducted from a separate electrical source. The influence of the magnetization system on the acceleration process is carried out through an electromagnetic field.

Modeling of processes occurring in accelerators with external augmenting systems is characterized by the fact that a subregion $G_{1}$, containing conducting bodies (guide rails, augmenting rails, an projectile) is incoherent (including the one across the boundary $\gamma_{12}$ ).

The study of the Maxwell system of equations in the quasistationary approximation in inhomogeneous regions with a connected conducting subregion with different ways of specifying boundary conditions was carried out in $[1,14,15]$, it was shown that the incoherence of the union of the conducting subregions and the boundary $\gamma_{12}$ can lead to nonuniqueness of the solution, i.e., the operator kernel will be non-empty. In this case, the magnetic field intensity vector $\mathbf{H}$ is uniquely determined both in the conductor and in the dielectric. The problem of a single solution separation arises only in the dielectric part in determining the electric field $\mathbf{E}$ (and potential $\mathbf{A}$ ). The dimension of the operator's kernel $\mathcal{M}$ is equal to the number of connected components of the conducting subregion and the boundary $\gamma_{12}$, and the basis vectors of the kernel have nonzero components only in the dielectric [12]. It should be noted that in [12] two main approaches to the search for the kernel of the operator task were considered.

Method of "fictional armature" introducing. It is clear from physical considerations that the considered process has unambiguous characteristics, and consequently, the formulation of a mathematical problem used in the process modeling should provide a unique solution. To obtain a unique solution of a system with a degenerate matrix, a normal solution can be found $[1,12,16]$, i.e., a solution with a minimal norm. This solution belongs to the space orthogonal to the kernel of the operator $\mathcal{M}[17,18]$.

To find a normal solution of a system with matrix $M$, there are methods [12], most of which require knowledge of the basis of the operator kernel. However, there are iterative methods for solving equations with a degenerate operator that do not require knowledge of the kernel [17].

Firstly it is necessary to find the kernel of the problem operator, i.e., to solve an auxiliary problem, then change the matrix of the system of linear algebraic equations taking into account knowledge of the kernel during the implementation most of the methods for finding a normal solution of a system of linear algebraic equations. As a rule, the constructed matrix is densely filled, and as a result the task of solving a system of linear equations 
M.P. Galanin, D.L. Sorokin

becomes more time-consuming. Iterative methods for finding a normal solution have a low rate of convergence, so it is impractical to use them for solving a non-stationary task.

An alternative way to solve the task is to introduce additional elements that transform a multiply-connected region into a simply-connected, but affect the electromagnetic field in the investigated area weakly [19].

The following connectivity components of the conducting subregion can be marked in the considered task: one component consists of guide rails, projectile, symmetry plane $(x=0)$ and side surface (ideal casing), others are formed by augmenting rails (the number of additional connected components is equal to the number of augmenting rails).

Let each additional element (hereinafter referred as "fictional armature") connect one connectivity component (one augmenting rail) with an ideal casing. The calculation of the electromagnetic field in the element will be carried out taking convective terms (as in the main projectile) into account:

$$
4 \pi \sigma\left\{[\mathbf{v} \times \operatorname{rot} \mathbf{A}]-\frac{D \mathbf{A}}{D t}+(\mathbf{v}, \nabla) \mathbf{A}\right\}=\operatorname{rot} \operatorname{rot} \mathbf{A} \text {. }
$$

The total current flowing through the "fictional armature" had to be equal to zero. This corresponds to the flow of electric current along the augmenting rails from one end of the computational domain to another. From the standpoint of mathematics, this is ensured by setting the condition $\mathbf{A}_{\tau}=0$ on the lateral surface of the computational region. It should be noted that the model of an ideal casing also requires setting $\mathbf{A}_{\tau}=0$, i.e., the introduction of a "fictional armature" does not complicate the procedure of setting boundary conditions. In addition, this method does not require additional computational costs and does not complicate the algorithm by solving secondary tasks. It is enough to make appropriate changes in the geometry of the computational redion for its implementation.

Application of the method of introduction of the "fictional armature". To demonstrate the method, we will perform the calculation of the electromagnetic field in the accelerator (Fig. 2, a). Due to the symmetry of the device, the simulation can be carried out for a quarter of the accelerator and the calculated area can be selected (Fig. 2, $b$ ).

We'll get a singular matrix $M$ when we try to solve a problem without using the "fictional armature" method and methods for a normal solution separation. So, the system of linear algebraic equations (for determinacy with 113257 unknowns) cannot be solved using the conjugate gradient method (the required accuracy is not achieved for over 1000 iterations). After the introduction of the 


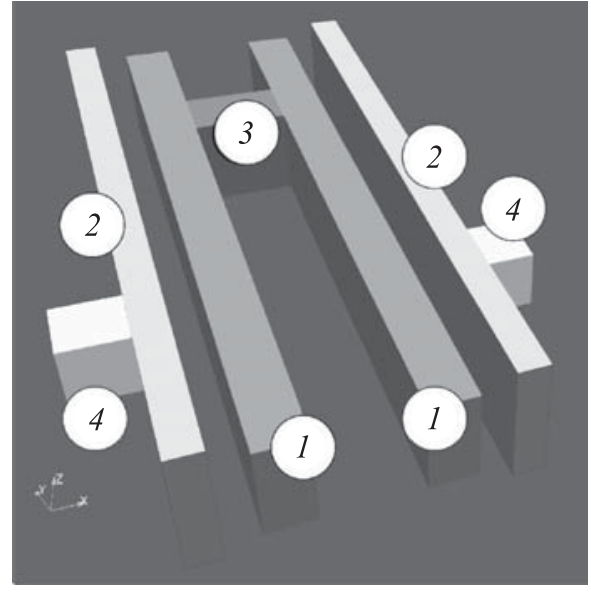

$a$

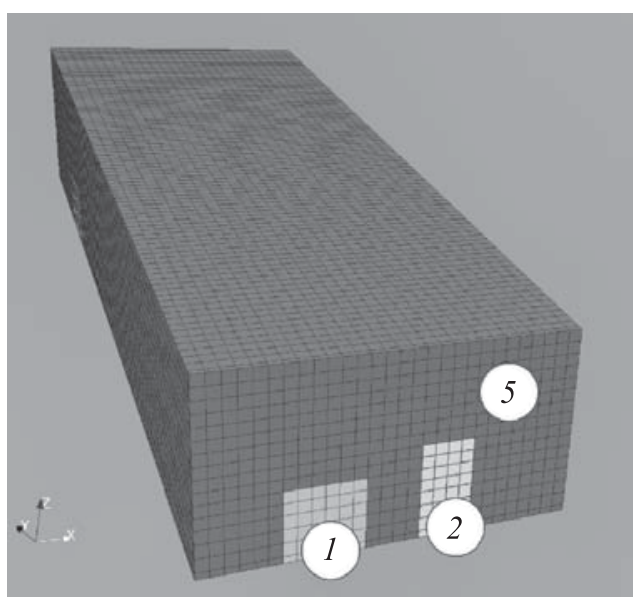

$b$

Fig. 2. The geometry of the device after the introduction of the "fictional armature"

(a) and the computational domain $(b)$ :

1 - main rails; 2 - augmenting rails; 3 - the main projectile; 4 - "fictional armature"; 5 - dielectric

"fictional armature", the method converges in 43 iterations, and the residual norm is $10^{-13}$.

Study of the computational domain size impact on the result. During the acceleration of bodies in the railgun, the most complex and interesting phenomena occur in the vicinity of the projectile, the characteristic length of which is comparable with the transverse size of the channel. So it is expedient to limit the considered space and to describe the fields in the spatial domain rigidly connected with the sliding projectile [1].

The problem of setting boundary conditions at the ends of the short computational domain arises with such approach, which allow to take into account the total currents flowing through the end sections of the rails. One of the ways to solve it is to specify the tangential components of the magnetic field strength vector $\Psi_{\tau}$, corresponding to the field around infinite conductors with the necessary currents at the ends of the region as the boundary conditions. The electromagnetic field required for $\Psi_{\tau}$, determination is the solution of a special spatial-two-dimensional task $[12,14,20]$.

The model has disadvantages. In particular, the current flow pattern near the $y$ boundaries (see Fig. 1) of the considered moving region does not correspond to the picture in the initial accelerator [14].

In order for edge effects not to affect significantly on the processes occurring in the vicinity of the projectile, the longitudinal size of the computational 
M.P. Galanin, D.L. Sorokin

domain must be noticeably larger than the characteristic dimensions of the projectile (in particular, the accelerator caliber. With a decrease in the computational domain length (size in the direction of the body overhang), a decrease in the calculated force acting on the projectile is observed, and, as a result, a decrease in the calculated muzzle velocity of the macrobody. This fact is confirmed by the results of a series of calculations of an accelerator without augmenting rails (Fig. $3, a$ ).
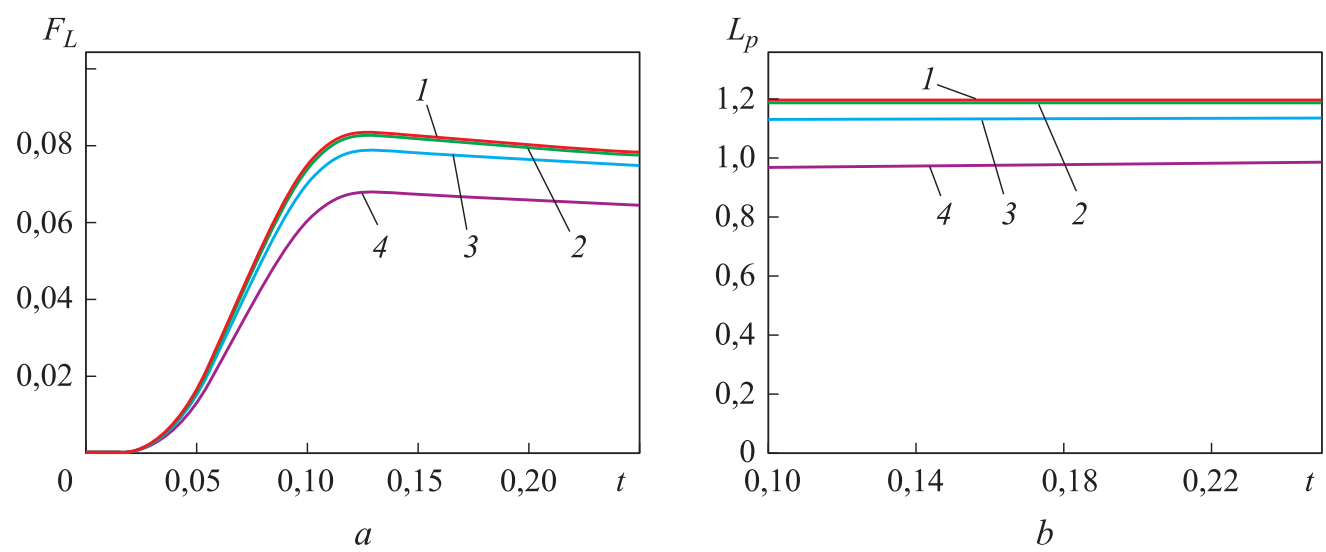

Fig. 3. Dependences of Lorentz force $(a)$ and linear inductance $(b)$ on time when calculating the acceleration of a body in an accelerator without augmenting rails with different lengths of the computational region:

$1-8$ calibers for the projectile; $2-4$ calibers for the projectile; $3-2$ calibers for the projectile; $4-1$ caliber for the projectile

In the process of acceleration, the linear inductance of the accelerator $L_{p}$ [21], determined from the ratio:

$$
F_{L}=\frac{L_{p} I^{2}}{2}
$$

is constant with high accuracy ( $F_{L}$ is Lorentz accelerating force; $I$ is current flowing through the guides). According to the results of computational experiments, the longer the patch region is, the better is the criterion for the constant inductance per unit (Fig. 3, b). It should be noted that the removal of the boundary $y=0$ of the computational domain from the projectile by more than 8 calibers does not have a noticeable effect on the quality of the result, but significantly increases the computational complexity.

The study of the influence of the "fictional armature" position on the result. The "fictional armature" should be at a sufficient distance from 
the main projectile in place not to affect the magnetic field in the vicinity of the main projectile. It is removed by 4 calibers in the demo calculation. To identify the degree of influence of the "fictional armature" position on the result of acceleration, several computational experiments were carried out, which showed that the approaching of the "fictional armature" to the main projectile leads to a (insignificant) decrease of the running inductance (Fig. 4). It should be noted that the position of the "fictional armature" relative to the main projectile affects the integral acceleration characteristics less than the change in the size of the computational domain in the $y$ direction. This proves the correctness of the method.
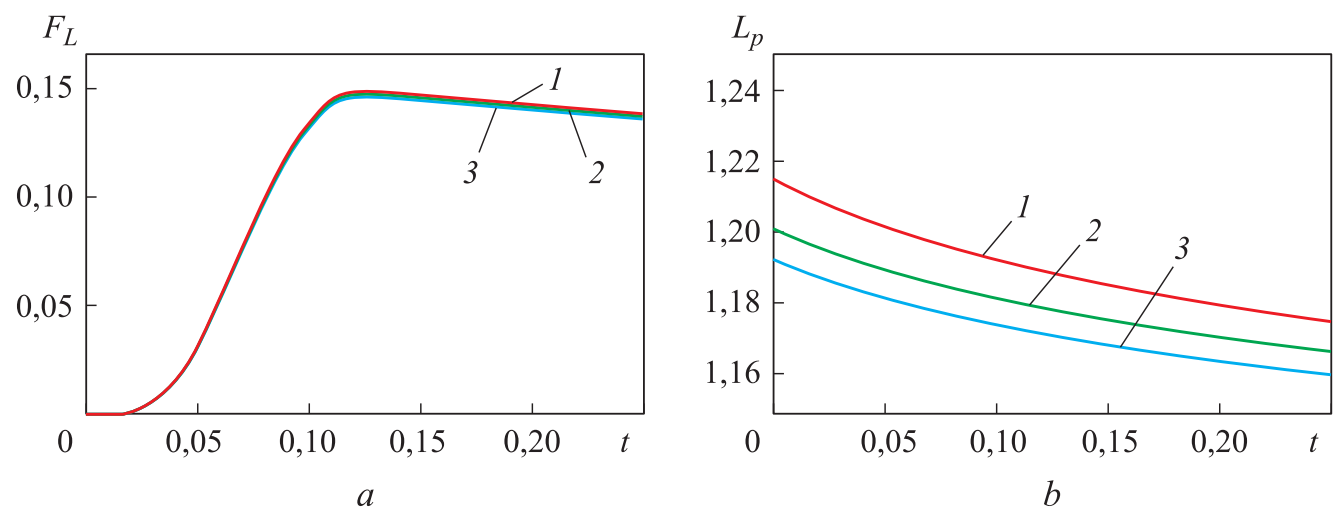

Fig. 4. Dependences of the Lorentz force $(a)$ and the linear inductance $(b)$ on time when calculating the acceleration of a body in an accelerator, shown in Fig. 2, $a$, with a different position of the "fictional armature":

1 - at a distance of 4 calibers; 2 - at a distance of 2 calibers; 3 - at a distance of 1 caliber from the projectile

Investigation of the effect of "fictional armature" electrical conductivity on the result. There is a possibility to vary not only the position and dimensions of the additional element, but also its conductivity, which is set factitiously, with supplementing the initial design of the device by "fictional armature". The results of a series of computational experiments with input data that differ from each other only by the values of the "fictional armature" indicate that the linear inductance in computational experiments practically does not depend on the conductivity of the "fictional armature" (Fig. 5).

Comparison of results obtained by the method of "fictional armature" and by the method of finding a normal solution of a system of linear algebraic equations. An electrodynamic accelerator was chosen for method comparison (Fig. 6, a). A mesh with crowding in the vicinity of conductors was introduced 

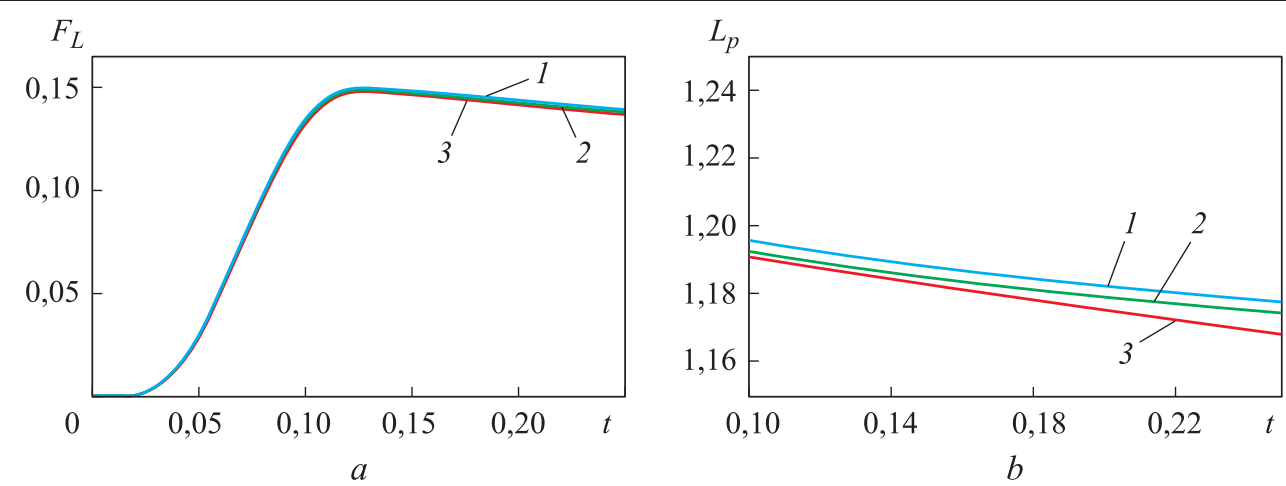

Fig. 5. Dependences of the Lorentz force $(a)$ and the linear inductance $(b)$ on time when calculating the acceleration of a body in an accelerator, shown in Fig. 2, $a$, with different values of the "fictional armature" conductivity

( $\sigma-$ conductivity of the augmenting rail material):

$$
1-\sigma / 10 ; 2-\sigma ; 3-10 \sigma
$$

in the region (Fig. 6, b) because it was required to solve systems of linear algebraic equations with strongly filled matrices (during the performing computational experiments, the proportion of nonzero elements in matrices was about $30 \%$ ) to find a normal solution. This allowed to reduce the number of unknowns.

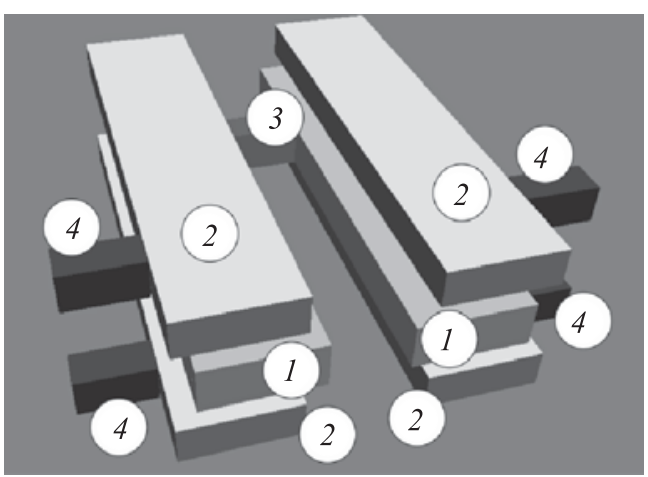

$a$

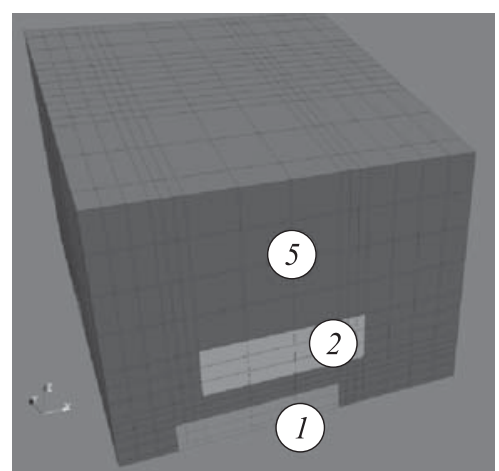

$b$

Fig. 6. The geometry of the device (a) and the computational domain (b):

1 - main rails; 2 - augmenting rails; 3 - the main projectile; 4 - "fictional armature"; 5 - dielectric

The results (Fig. 7) indicate that the discrepancy between the two methods in determining the accelerating force is $0.5 \%$. A comparison of the local acceleration characteristics, in particular, the distributions of the magnetic field strength vector in the accelerator channel at time 0.058 shows that the results obtained by the two methods differ by no more than $3 \%$. 

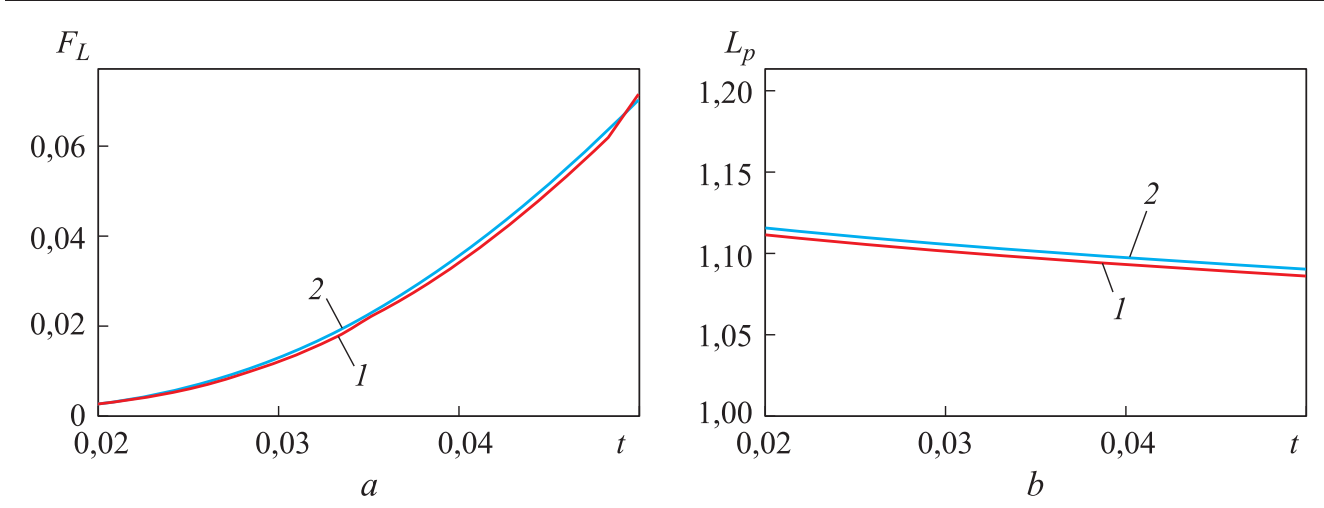

Fig. 7. Dependences of Lorentz force ( $a$ ) and running inductance $(b)$ on time when calculating acceleration using the "fictional armature" method (1) and the method of the normal solution retrieving (2)

Conclusion. The features of solving the Maxwell system of equations in the MHD-approximation while modeling the process of a macrobody acceleration in a rail accelerator with a augmenting system are described. It is noted that in the considered case, the computational domain contains several disconnected conducting subregions. The methods for obtaining a single solution of the task were analyzed.

A method of solution, consisting in the implementation of a "fictional armature", has been developed. The proposed method has the lowest computational complexity among analyzed ones. The performed computational experiments (calculations of the acceleration process) showed the method robustness.

\section{REFERENCES}

Translated by E. Ovsyannikova

[1] Galanin M.P., Popov Yu.P. Kvazistatsionarnye elektromagnitnye polya v neodnorodnykh sredakh [Quasistationary electromagnetic fields in heterogeneous medium]. Moscow, Nauka Publ., Fizmatlit Publ., 1995.

[2] Galanin M.P., Lototskii A.P., Popov Yu.P., et al. Three-dimensional phenomena at electromagnetic launch of conducting armatures. Matem. mod., 1999, vol. 11, no. 8, pp. 3-22 (in Russ.).

[3] Galanin M.P., Lototskiy A.P., Urazov S.S., et al. The mathematical simulation of metallic contacts erosion in railgun accelerator. Preprinty IPM im. M.V. Keldysha RAN [Preprints of the Keldysh Institute of Applied Mathematics], 2003, no. 79 (in Russ.).

[4] Roch M. Experimental investigation of augmented electromagnetic accelerators. Dissertation zur Erlangung des akademischen Grades eines Doktor der Ingenieurwissenschaften. Universität Kassel, 2016. 
M.P. Galanin, D.L. Sorokin

[5] Babakov Y.P., Plekhanov A.V., Zheleznyi V.B. Range and railgun development results at LS\&PA "SOYUZ". IEEE Trans. Magn., 1995, vol. 31, iss. 1, pp. 259-262. DOI: $10.1109 / 20.364691$

[6] Coffo M. Contribution a la modelisation, a l'optimisation et a l'etude experimentale d'un lanceur a rails augmente et du projectile. These pour obtenir le grand de docteur. Autre. Universite de Franche-Comte, 2011. Francais.

[7] Kondratenko A.K., Poltanov A.E., Ryndin V.N., et al. Analysis of some variants of multirail systems with close magnetic coupling. Preprint TRINITI, 1998, no. 0047-A (in Russ.).

[8] Glinov A., Poltanov A., Kondratenko A. Comparison analysis of processes occurring in $\mathrm{N}$-turn and classical railguns. High Temp., 2007, vol. 45, iss. 3, pp. 298-304. DOI: 10.1134/S0018151X07030030

[9] Milekhin Yu.M., Kononov B.V., Syrtsov E.B., et al. Composition conception of multiturn rail accelerator and their implementation. Izvestiya vuzov. Fizika, 2013, vol. 56, no. 6-3, pp. 42-44 (in Russ.).

[10] Watt T., Crawford M. Experimental results from a two-turn $40 \mathrm{~mm}$ railgun. IEEE Trans. Magn., 2009, vol. 45, iss. 1, pp. 490-494. DOI: 10.1109/TMAG.2008.2008872

[11] Kulikovskiy A.G., Lyubimov G.A. Magnitnaya gidrodinamika [Magnetic hydrodynamics]. Moscow, Fizmatgiz Publ., 1962.

[12] Urazov S.S. Matematicheskoe modelirovanie mnogomernykh kvazistatsionarnykh elektromagnitnykh poley v kanale elektrodinamicheskogo uskoritelya. Dis. kand. fiz.-mat. nauk [Mathematical simulation of multidimensional quasistationary fields in electromagnetic rail gun channel. Cand. Phys.-Math. Sc. Dis.]. Moscow, 2007 (in Russ.).

[13] Samarskii A.A., Tishkin V.F., Favorski A.P., et al. Operator-difference schemes. Differ. uravn., 1981, vol. 17, no. 7, pp. 1317-1327 (in Russ.).

[14] Galanin M.P. Computer modelling in problems of electromagnetic and kinetic energy conversion. Problems and models. Informatsionnye tekhnologii i vychislitel'nye sistemy [Journal of Information Technologies and Computing Systems], 2002, no. 4, pp. 109-123 (in Russ.).

[15] Galanin M.P. Computer modelling in problems of electromagnetic and kinetic energy conversion. Reshenie zadach. Informatsionnye tekhnologii i vychislitel'nye sistemy [Journal of Information Technologies and Computing Systems], 2003, no. 1-2, pp. 112-127 (in Russ.).

[16] Samarskiy A.A., Popov Yu.P. Raznostnye metody resheniya gazovoy dinamiki [Differential methods of gas dynamics]. Moscow, Editorial URSS Publ., 2004.

[17] Samarskiy A.A., Nikolaev E.S. Metody resheniya setochnykh uravneniy [Solution technique for finite-difference equations]. Moscow, Nauka Publ., 1978.

[18] Faddeev D.K., Faddeeva V.N. Vychislitel'nye metody lineynoy algebry [Computational methods of computer algebra]. Moscow, Fizmatgiz Publ., 1963.

14 ISSN 1812-3368. Вестник МГТУ им. Н.Э. Баумана. Сер. Естественные науки. 2019. № 1 
Simulation of Quasistationary Electromagnetic Fields in Regions...

[19] Galanin M.P., Sorokin D.L. Modelling of quasistationary electromagnetic fields in regions with disconnected conductive subregions. Preprinty IPM im. M.V. Keldysha RAN [Preprints of the Keldysh Institute of Applied Mathematics], 2017, no. 19 (in Russ.).

[20] Nikol'skiy V.V., Nikol'skaya T.I. Elektrodinamika i rasprostranenie radiovoln [Electrodynamics and waves propagation]. Moscow, Nauka Publ., 1989.

[21] Kalantarov P.L., Tseytlin L.A. Raschet induktivnostey [Calculation of inductances]. Leningrad, Energoatomizdat Publ., 1986.

Galanin M.P. - Dr. Sc. (Phys.-Math.), Professor, Head of the Department, Keldysh Institute of Applied Mathematics, Russian Academy of Sciences (Miusskaya ploshad 4, Moscow, 125047 Russian Federation), Professor, Department of Applied Mathematics, Bauman Moscow State Technical University (2-ya Baumanskaya ul. 5, str. 1, Moscow, 105005 Russian Federation).

Sorokin D.L. - Assistant, Keldysh Institute of Applied Mathematics, Russian Academy of Sciences (Miusskaya ploshad 4, Moscow, 125047 Russian Federation).

\section{Please cite this article as:}

Galanin M.P., Sorokin D.L. Simulation of Quasistationary Electromagnetic Fields in Regions Containing Disconnected Conducting Subregions. Herald of the Bauman Moscow State Technical University, Natural Sciences, 2019, no. 1, pp. 4-15.

DOI: 10.18698/1812-3368-2019-1-4-15 